\title{
The Improvement of Telerobotic System Behavior in Contact with Remote Environment by Extension of an Impedance Controller
}

\author{
doi:10.3991/ijoe.v4i4.698 \\ J. Velagic, S. Huseinbegovic and N. Osmic \\ Faculty of Electrical Engineering, Sarajevo, Bosnia and Herzegovina
}

\begin{abstract}
The teleoperation (telerobotic) systems often face two key challenges: the existence of communication delays between the master and slave site as well as the addition of force feedback to improve the user's sense of presence. The first goal of this paper is that the slave manipulator should track the position of the master manipulator and the second goal is that the environmental force acting on the slave, when it contacts a remote environment, be accurately transmitted to the master. For solving both problems we proposed the symmetric impedance matched teleoperation systems with a wave filter in feedback loop. Simulations results using a single-degree of freedom master/slave system are presented showing the performance of the resulting system.
\end{abstract}

Index Terms-telerobotics, telerobotics, master-slave concept, remote control, impedance controller, position tracking

\section{INTRODUCTION}

Teloperation systems have been popular for decades especially for hazardous and unstructured tasks. Such tasks include nuclear reactors, space applications, military uses, medical operations and deep-sea explorations to name a few [1], [2]. More recently, explorations on Mars have renewed the interest in this topic.

Teleoperation allows users to manipulate and interact with environments via master and slave robotic mechanisms. This enables operation at a distance and can also scale human forces and motion to achieve stronger, bigger, or smaller interfaces. The information sent from the master to the slave controller is the position and/or velocity command and the information sent from the slave to the master is usually the force command. The force feedback from the slave to the master, representing contact information, provides a more extensive sense of telepresence [3]. When this is done the teleoperator is said to be controlled bilaterally.

In bilateral teleoperation, the master and the slave manipulators are coupled via a communication network and time delay is incurred in transmission of data between the master and slave sites. It is well known that the delays in a closed loop system can destabilize an otherwise stable system [4]. Time delay instability in force reflecting teleoperation was a long standing impediment to bilateral teleoperation with force feedback. To avoid this problem many concepts, such as Network Theory, Passivity and Scattering Theory were used to analyze mechanisms responsible for loss of stability and derive a time delay compensation scheme to guarantee stability independent of the (constant) delay [4], [5], [6] and [7].

All mentioned methodologies work very well under assumptions that master and slave robots move in free space [6]. When remote environment act on the slave robot the control performance will be violated. The most widely used techniques for this problem solving are based on investigation of a Smith-like prediction within the remote site [7].

In our paper we applied a wave filter in an impedance matching teleoperation system with the aim to improved tracking performance under contact with remote environment. Beside the wave filter reduces wave reflections it also smoothes the system behavior regardless of operating conditions or task. Also, inserting a filter into the wave transmission path between master and slave site does not affect passivity. The effect of using the wave filter into impedance matching teleoperation system will be demonstrated.

\section{SYSTEM DESCRIPTION}

The proposed impedance matching control system with the wave filter in a master feedback loop is shown in Fig. 1. This system contains a symmetric configuration with impedance controllers at both sites (master and slave). The master and slave robots are presented as single degree of freedom manipulators. More specifically, both impedance controllers accept a velocity command from the incoming waves and return forces.

Also note the introduction of two dissipation elements in both impedance controllers: $R$ and $D$. The damping $D$ is used to create a critically damped response, necessary for the dynamic simplification. The damping $R$ is added to match the plain controller impedance to the wave impedance and help dissipate the communications resonance if needed.

The wave communications element acts as a true admittance: it connects the master and slave impedances, observes forces on both sides and updates the desired motions as needed. It is the stiffness of the communications. This classical impedance matched system is capable to eliminate all returning waves (wave reflections) under the assumption that both robots move as pure inertias in free space. When contact with remote environment is made, this model is no longer valid, and the returning wave signals carry the contact information. In this case, the control performance can be improved by including a wave filter in the master feedback loop. 


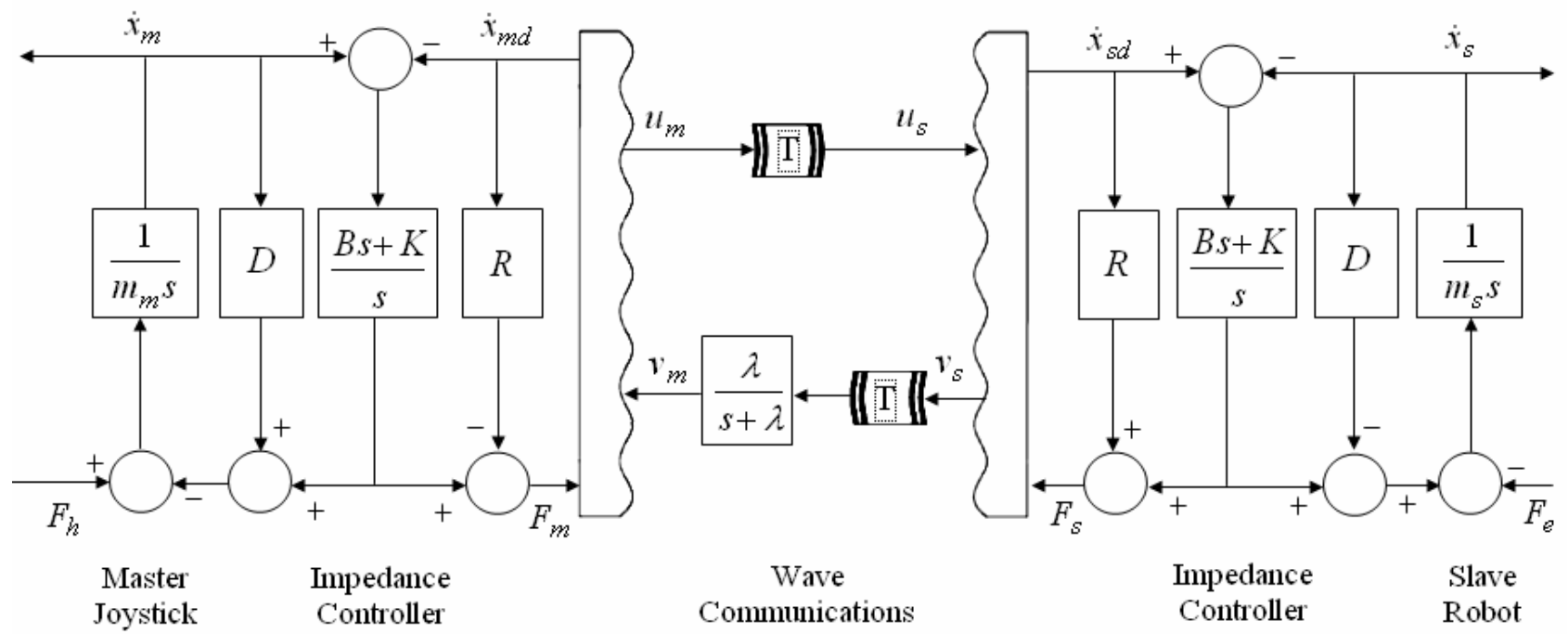

Figure 1. The symmetric impedance matched teleoperation system with the wave filter.

The components of proposed teleoperation system will be shortly described in the rest of this section.

\section{A. Master and Slave Manipulators}

Position figures and tables at the tops and bottoms of columns. Avoid placing them in the middle of columns. Large figures and tables may span across both columns. Figure captions should be centered below the figures; table captions should be centered above. Avoid placing figures and tables before their first mention in the text. Use the abbreviation "Fig. 1," even at the beginning of a sentence.

Master and slave manipulators motion equations are:

$$
\begin{gathered}
J_{m}+b_{m} \stackrel{\&}{\&}=F_{m}, \\
J_{s}+b_{s} \cdot \underset{s}{\&}=F_{s} .
\end{gathered}
$$

where $J$ is inertia moment, $b$ is damping factor, $k$ is coefficient of elasticity, \& and are segment velocity and acceleration, respectively, and $F$ is moment of rotation. We denote the master variables at the local site with subscript " $m$ " and the slave variables at the remote location with " $s$ ".

The tracking position is obtained using PD controller:

$$
\begin{aligned}
& F_{p d}=K\left(x_{m}-x_{s}\right)+B\left(\&_{m}-\&_{s}\right) \\
& F_{m}=-F_{p d}, F_{s}=F_{p d}
\end{aligned}
$$

where $K$ and $B$ are proportional and derivate coefficients of PD controller.

Traditional force reflecting teleoperators transmit force and position/velocity commands between the master and slave sites. Untreated, they experience stability problems when the transmission involves even small time delays. These problems have been solved by using wave variables [4], [5].
Note that the delays in the forward and reverse path need not be the same, although the following derivations make this assumption to simplify notation. However, for this analysis to be applicable both delays should remain constant and add up to $2 T$.

\section{B. Wave transformations and communications}

Wave variables present a modification or extension to the theory of passivity which creates robustness to arbitrary time delays. This suggests performing the communications directly in the wave domain, as shown in Fig. 1.

Based only on the concepts of power and energy, they are applicable to nonlinear systems and can handle unknown models and large uncertainties. As such, they are well suited for interaction with real physical environments.

( A complementary pair of wave variable $(u, v)$ is defined based on a complementary pair of standard power variables $(x, F)$ by the following transformation or encoding

$$
\begin{aligned}
& u_{m}(t)=\frac{b{\underset{m}{\&}}_{k}(t)+F_{m}(t)}{\sqrt{2 b}} \\
& v_{m}(t)=\frac{b{ }_{m}^{\&}(t)-F_{m}(t)}{\sqrt{2 b}},
\end{aligned}
$$

and

$$
\begin{aligned}
& u_{s}(t)=\frac{b \&_{s d}(t)+F_{s}(t)}{\sqrt{2 b}} \\
& v_{s}(t)=\frac{b \&_{s d}(t)-F_{m}(t)}{\sqrt{2 b}} .
\end{aligned}
$$

We select $u$ to denote the forward or right moving wave, while $v$ denotes the backward or left moving wave. The characteristic wave impedance $b$ is a positive constant 
and assumes the role of a tuning parameter, trading off speed of motion against level of forces [6].

\section{Impedance matching system}

Wave reflections appear when a wave signal hits an element with impedance unequal to its own wave impedance $b$. So, to reduce reflections, we try to match the impedance for both master and slave subsystems. This is analogous to using master and slave energy dissipation as damping for the communications resonance.

Impedance matching can be decomposed into two steps. In the first step, both the master and slave subsystems are configured and tuned independently. Each appears as a pure damper to the wave commands, although their impedance value remains unspecified. This value is selected and adjusted in step two, according to the desired task.

In the next section a design of impedance controller will be considered.

\section{DESIGN OF IMPEDANCE CONTROLLER With WAVE FILTER}

The objective of impedance controller design is to obtained values of parameters $K, B, D$ and $R$ (see Fig. 1), which ensure that wave reflections will acquire minimal values.

The master side is described with the following equations in Laplace domain:

$$
\begin{aligned}
& s X_{m}=\frac{1}{J_{s}+b}\left[F_{h}-X\right] \\
& X=\left(D s X_{m}+(B s+K)\left(X_{m}-X_{m d}\right) \tilde{)} .\right. \\
& F_{m}=(B s+K)\left(X_{m}-X_{m d}\right)-R s X_{m d}
\end{aligned}
$$

Eliminating $X_{m}$ from above equations, we have:

$$
G_{N} \mathrm{~T}_{m}=G_{B 1} \mathrm{~T}_{h}-G_{B 2} s \Theta_{m d}
$$

where:

$$
\begin{aligned}
G_{N} & =J s^{2}+(b+B+D) s+K \\
G_{B 1} & =(B s+K) \\
G_{B 2} & =J(B+R) s^{2} \\
& +(J K+(B+R)(D+b)+R B) s \\
& +K(b+D+R)
\end{aligned}
$$

Using the same procedure for the slave side, the following equation is achieved:

$$
G_{N} F_{s}=G_{B 1} F_{e}+G_{B 2} s X_{s d} .
$$

The wave reflection will be eliminated if the following conditions are satisfied:

$$
\begin{aligned}
& G_{N}=b G_{B 2} \\
& G_{N}=\frac{\lambda_{s}}{s+\lambda_{s}} G_{B 1} .
\end{aligned}
$$

Solving equations (9) yields

$$
\begin{aligned}
& B=J \lambda_{s} \\
& K=J \lambda_{s}^{2} \\
& D=J \lambda_{s}-b, \\
& R=\frac{b}{J}-J \lambda_{s}
\end{aligned}
$$

where $\lambda_{s}$ is bandwidth.

Substituting (9) into (7) and (8) yields:

$$
\begin{aligned}
& F_{m}=\frac{\lambda_{s}}{s+\lambda_{s}} F_{h}-b s X_{m d} \\
& F_{s}=\frac{\lambda_{s}}{s+\lambda_{s}} F_{e}+b s X_{s d}
\end{aligned} .
$$

Using (11) equations (4) and (5) become:

$$
\begin{aligned}
& U_{m}=\frac{1}{\sqrt{2 b}} \frac{\lambda_{s}}{s+\lambda_{s}} F_{h} \\
& V_{s}=-\frac{1}{\sqrt{2 b}} \frac{\lambda_{s}}{s+\lambda_{s}} F_{e}
\end{aligned}
$$

From the equation (12) can be concluded that wave reflections will be eliminated in the case no external forces act on the slave manipulator.

For free space motions with no expected contact or other disturbance forces, reduce $b$ as far as possible to minimize damping and inertial effects and allow quick moves. During this task no wave responses or reflections will appear, and the user can work freely, as though there was no force feedback at all.

Finally notice the lower limit for the wave impedance $b$ from equation (10):

$$
b \geq B=J \lambda_{s} .
$$

To ensure impedance matching, the wave impedance must always be greater than or equal to the master or slave dissipation, because a passive controller can only add damping. If the lower limit on $b$ is too high, we have to retune the master/slave controllers to a lower bandwidth with lower dissipation requirements.

When we expect contact with the remote environment, we increase $b$. Higher values of $b$ provide more resistance to motion, which will prevent the manipulator from creating high impact forces. Also the increased steadystate stiffness ensures that contact forces are fed back to the master in close proximity to the actual contact location, providing good spatial resolution. 


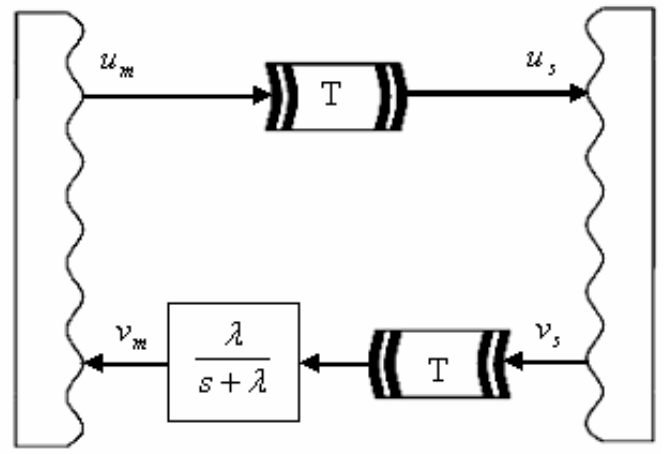

Figure 2. The communication wave channel with a wave filter.

The second solution is introducing the wave filter in the master force feedback loop (Fig 2.), which is used in this paper.

In control system theory a filter is used for reducing high-frequency noise. However, it introduces an additional time delay that increases risk of system instability. This has identical effect as a large dead time. The filter is not passive and can produces that system becomes unstable. Inserting a filter into the wave transmission path between master and slave sites does not affect passivity. Indeed the wave variables are constructed to be unaffected by delays or phase lag. The only requirement on the filters is that their gain must be limited below unity, so, for example, linear filters may not be underdamped. Filtering creates a smoother behavior by eliminating the high-frequency components often seen in the wave reflections. Generally the filter constants should be chosen such that the bandwidth is close to the actual time delay which dominates the closed-loop behavior. Wave filtering increases inertia and reduces stiffness, much like the pure delay. In this paper we used the first order filter. The average filter frequency is chosen depending on $T$, because a frequency of wave reflections is equal $1 / 2 T$. The recommended values for the wave filter are $\lambda \geq 1 / T$. The effect of wave filter on system performance will be demonstrated in next section.

\section{Simulation Results}

In these simulations, we consider a pair of one degree of freedom manipulators. We choose the following parameters for master and slave manipulators: $J_{m}=J_{s}=2 \mathrm{kgm}^{2}$ and $b_{m}=b_{s}=1 \mathrm{kgm}^{2} / \mathrm{s}$. The communication delay is $400 \mathrm{~ms}$ in both paths and bandwidth $\lambda_{s}$ is chosen to be 10 . From these values we obtained the following parameters of the impedance controller: $B=20 \mathrm{kgm} / \mathrm{s}^{2}$, $K=200 \mathrm{kgm} / \mathrm{s}, D=19$ i $R=-19.5$.

In addition we consider three different scenarios. In the first scenario, at the beginning $0-1 \mathrm{~s}$, the human operator acts on the master manipulator with the torque equal 10 $\mathrm{Nm}$ and there is no contact the slave manipulator with a remote environment.

The simulation results, which demonstrate position, velocity and torque for both master and slave manipulator, and consequently position and velocity differences between mentioned manipulators are shown in Figs. 3-6.
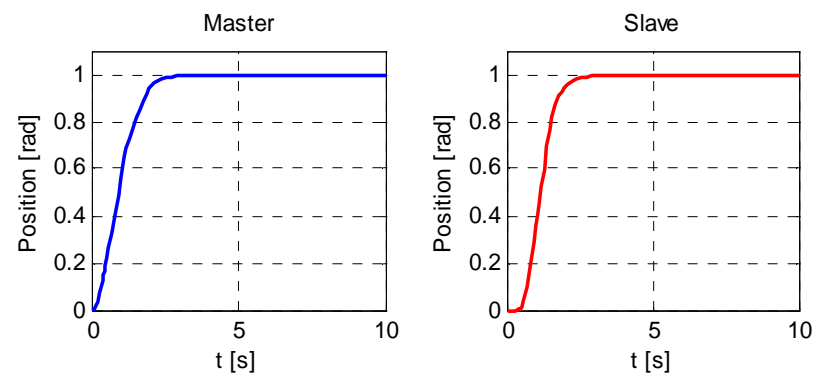

Figure 3. Positions of master and slave manipulators.
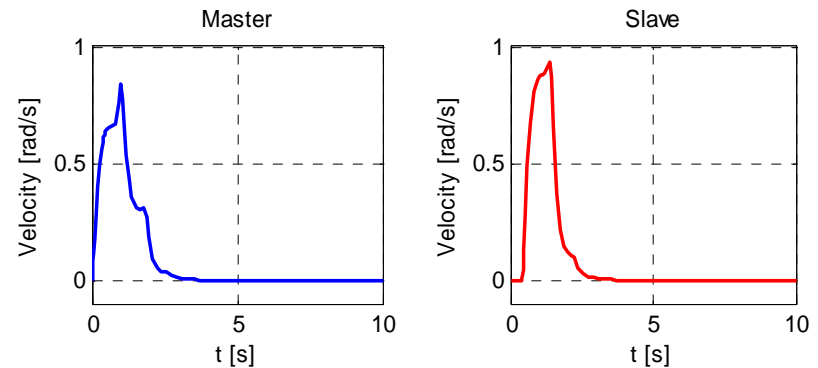

Figure 4. Velocities of master and slave manipulators.
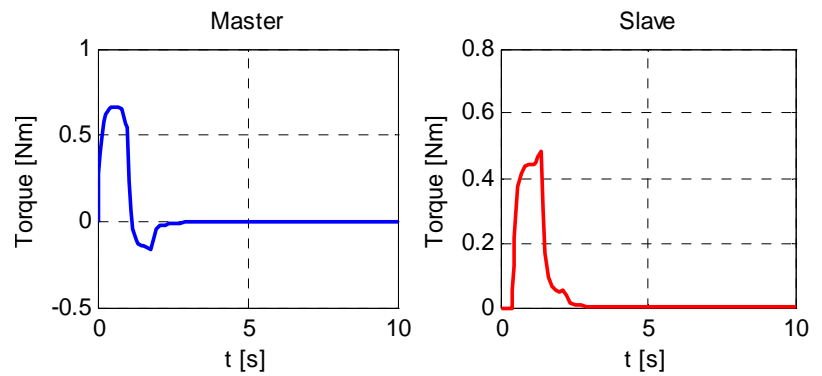

Figure 5. Torques of master and slave manipulators.
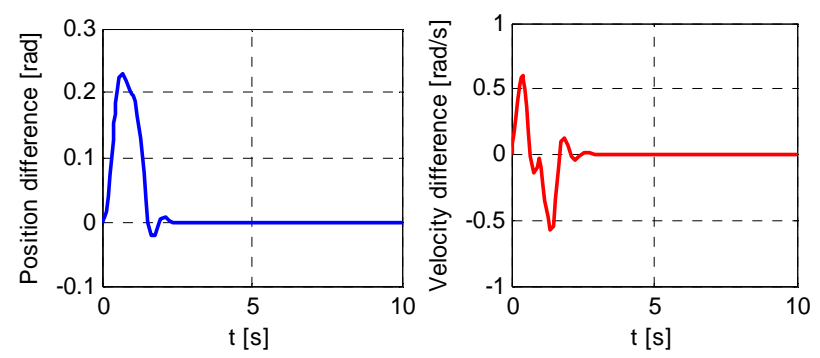

Figure 6. Position and velocity differences of master and slave manipulators.

The second scenario, the human operator acts on the master manipulator during the whole process with the 1 $\mathrm{Nm}$ torque. During the motion, the slave manipulator goes in a contact with the external environment (wall). This external force can be modeled in the way that slave velocity fall to zero when the angle exceeds an appropriate value. From the simulation results obtained (Figs. 7-10) can be concluded that tracking performance is violated. The influence of contact forces on the slave side is demonstrated by large values of torques in the master feedback (Fig. 9). 

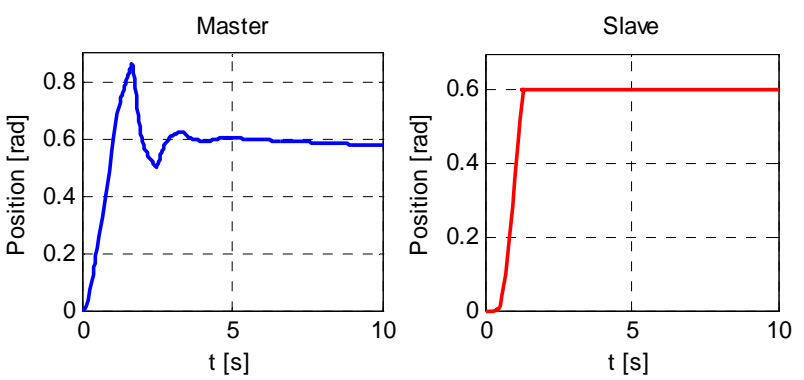

Figure 7. Master and slave positions when a force is applied at the slave side.
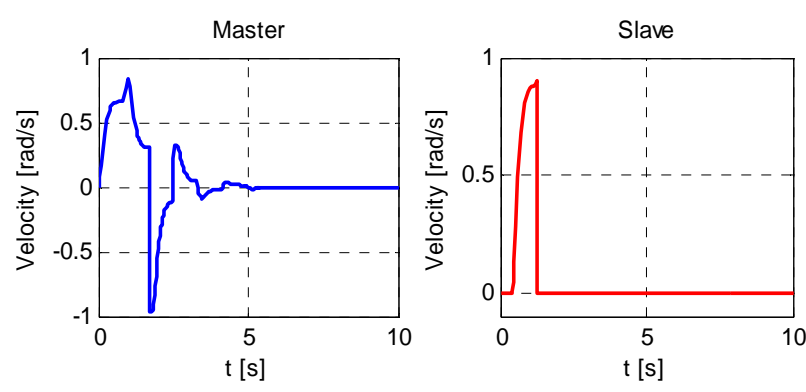

Figure 8. Master and slave velocities when a force is applied at the slave side.
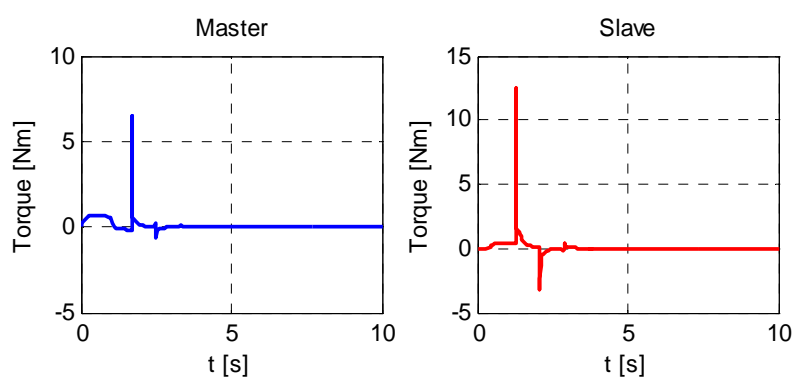

Figure 9. Master and slave torques when a force is applied at the slave side.
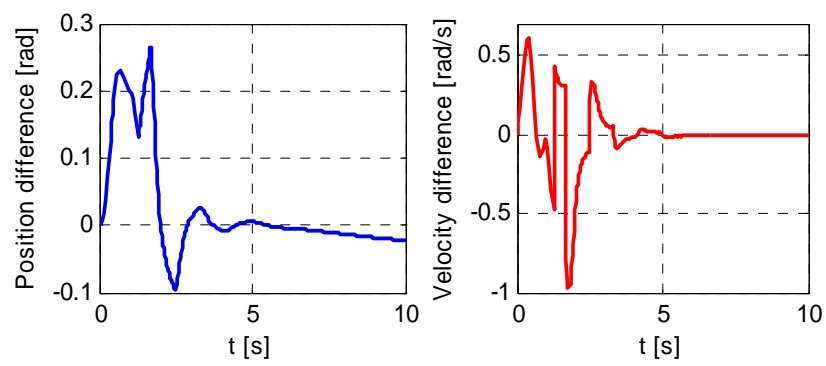

Figure 10. Position and velocity differences between master and slave manipulators when a force is applied at the slave side.

The implementation of the proposed impedance controller with wave filter, when external environment acts on remote slave manipulator, is considered in the third scenario. The objective is to improve the control performance during the contact. On the other words, a torque on the master side, which is result of a contact from the remote side, is needed to decrease. Also, the performance of master-slave position tracking is necessary to improved.

Simulation results illustrated the good position tracking with small communication delay (Fig. 11). The torque acts on the master manipulator is decreased about ten times (Fig. 13). Differences of position and velocity between master and slave manipulators are shown in Fig. 14, respectively. From simulation results obtained (Figs. 1114), can be concluded that satisfactory position and velocity tracing performance are achieved with simultaneously a master torque decreasing.

The results of comparative analysis between impedance controller with and without wave filter in the master feedback loop are illustrated in Figs. 15 and 16.
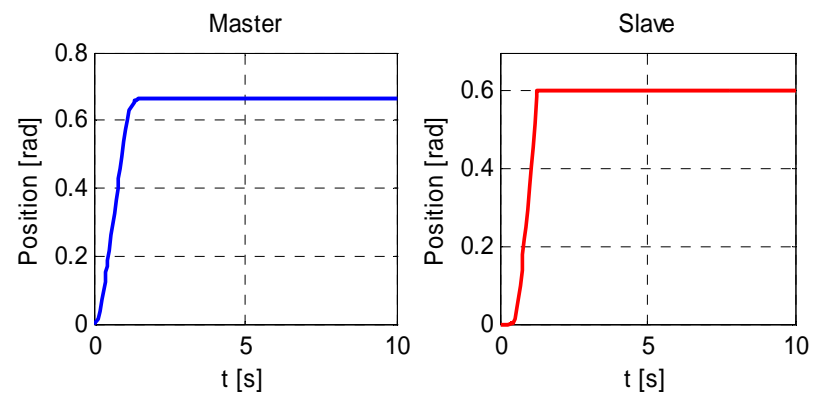

Figure 11. Master and slave positions when a force acts at the slave side using the impedance controller with wave filter.
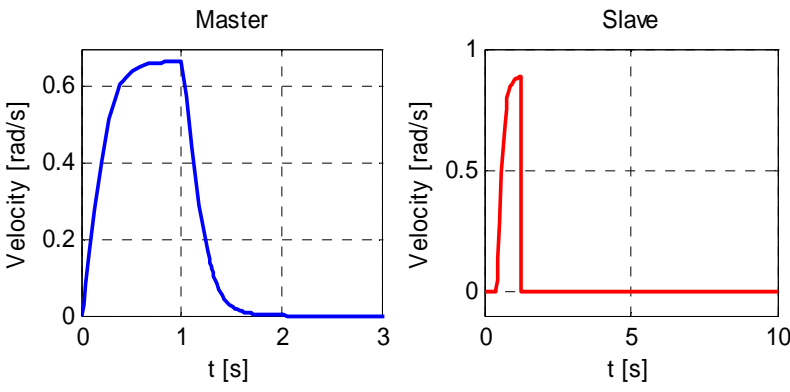

Figure 12. Master and slave velocities when a force acts at the slave side using the impedance controller with wave filter.
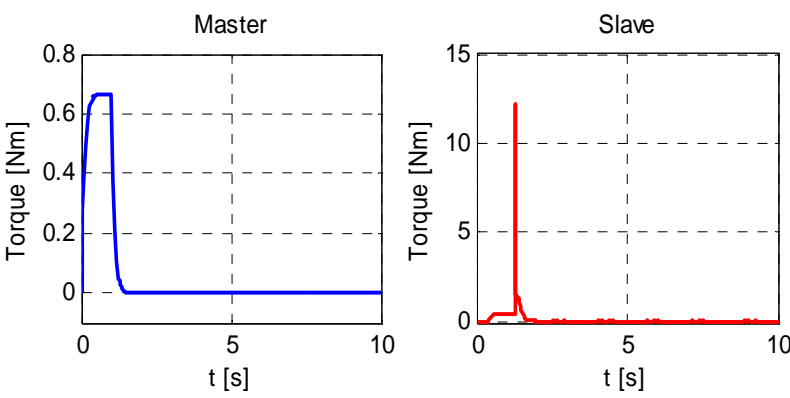

Figure 13. Torques of master and slave when a force acts at the slave side using the impedance controller with wave filter.
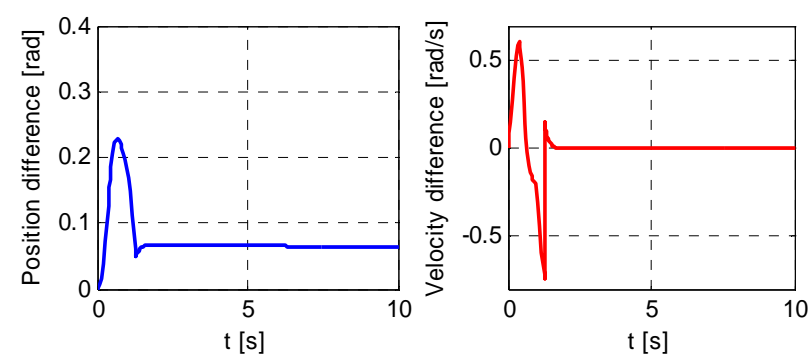

Figure 14. Position and velocity differences between master and slave manipulators when a force is applied at the slave side using the impedance controller with wave filter. 

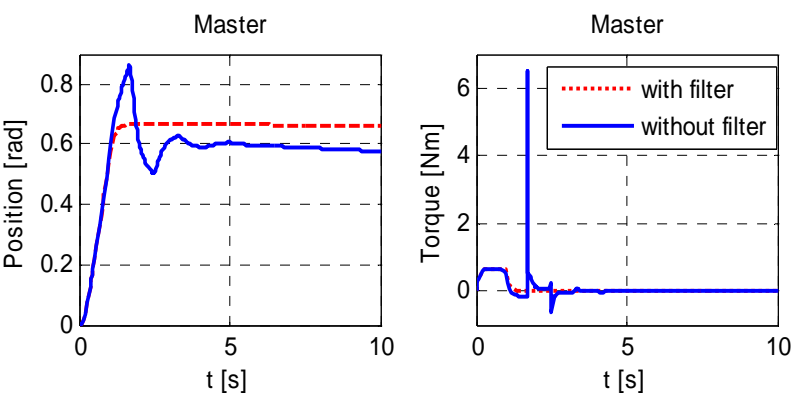

Figure 15. Positions and torques of master manipulator with and without wave filter in master feedback loop.
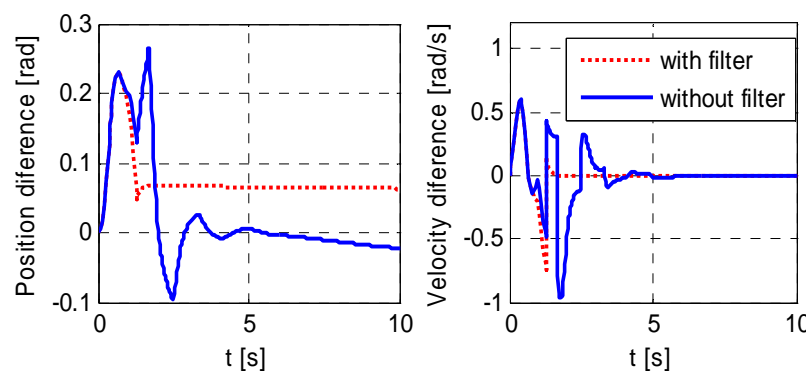

Figure 16. Position and velocity differences between master and slave manipulators with and without wave filter in master feedback loop.

\section{CONCLUSIONS}

In this paper we presented the extension of the impedance matched teleoperation system by using the wave filter placed in a master feedback. The impedance matched teleoperation system demonstrates satisfactory position, velocity and torque tracking performance in presence a significant communication delay in the system. The performance of this system is violated in the case an external force is applied at the slave manipulator side. For improving control performance when an external force is applied at remote master slave side we introduced the wave filter in master feedback which decreases the torque acts on master manipulator. The simulation results show that proposed teleoperation control system is robust and produces good position, velocity and tracking capabilities.

\section{REFERENCES}

[1] C. Kitts, "Development and Teleoperation of Robotic Vehicles," AIAA Unmanned Unlimited Systems, Technologies and Operations Conference, San Diego, California, 2003.

[2] T. B. Sheridan, "Space teleoperation through time delay: Review and prognosis," IEEE Transactions on Robotics and Automation, vol. 9, no. 5, pp. 592-606, October 1993. (doi:10.1109/70.258052)

[3] T. B. Sheridan, Telerobotics, Automation, and Human Supervisory Control, Cambridge: MIT Press MA, 1992.

[4] R.J. Anderson, and M.W. Spong, "Bilateral Control of Teleoperators with Time Delay," IEEE Transactions on Automatic Control, vol. 34, no. 5, pp. 494-501, May 1989. (doi:10.1109/9.24201)

[5] G. Niemeyer, and J. Slotine, "Stable Adaptive Teleoperation," International Journal on Oceanic Engineering, vol 16, pp 152-162, 1991. (doi:10.1109/48.64895)

[6] G. Niemeyer, and J. Slotine, "Telemanipulation with Time Delays," International Journal of Robotics Research, vol. 23, no. 9, pp. 873-890, 2004. (doi:10.1177/0278364904045563)

[7] Y. Yokokohji, T. Imaida, and Y. Yoshicawa, "Bilateral Teleoperation under Time-varying Communication Delay," IEEE/RSJ International Conference on Intelligent Robots and Systems, pp. 1854-1859, 1999.

[8] D. Lee, and M.W. Spong, "Passive Bilateral Teleoperation with Constant Time Delay," IEEE Transactions on Robotics and Automation, vol. 22, no.2, pp. 269-281, April 2006.

\section{AUTHORS}

J. Velagic is with the University of Sarajevo, Faculty of Electrical Engineering, Department of Automatic Control and Electronics, Sarajevo, Bosnia and Herzegovina (email: jasmin.velagic@etf.unsa.ba).

S. Huseinbegovic is with the University of Sarajevo, Faculty of Electrical Engineering, Department of Automatic Control and Electronics, Sarajevo, Bosnia and Herzegovina (e-mail: senad.huseinbegovic@etf.unsa.ba).

N. Osmic is with the University of Sarajevo, Faculty of Electrical Engineering, Department of Automatic Control and Electronics, Sarajevo, Bosnia and Herzegovina (email: nedim.osmic@etf.unsa.ba).

This work was supported in part by the Cantonal Ministry of Science and Education, Sarajevo. Manuscript received 18 June 2008. Published as submitted by the authors. 